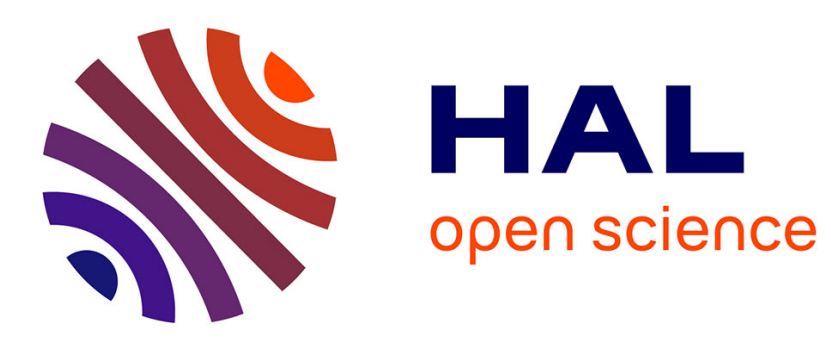

\title{
Analysis, simulation and impedance operator of a nonlocal model of porous medium for acoustic control Emmanuel Montseny, Céline Casenave
}

\section{To cite this version:}

Emmanuel Montseny, Céline Casenave. Analysis, simulation and impedance operator of a nonlocal model of porous medium for acoustic control. Journal of Vibration and Control, 2015, 21 (5), pp. 1012-1028. 10.1177/1077546313493815 . hal-00854312

\section{HAL Id: hal-00854312 \\ https://hal.science/hal-00854312}

Submitted on 26 Aug 2013

HAL is a multi-disciplinary open access archive for the deposit and dissemination of scientific research documents, whether they are published or not. The documents may come from teaching and research institutions in France or abroad, or from public or private research centers.
L'archive ouverte pluridisciplinaire HAL, est destinée au dépôt et à la diffusion de documents scientifiques de niveau recherche, publiés ou non, émanant des établissements d'enseignement et de recherche français ou étrangers, des laboratoires publics ou privés. 


\title{
Analysis, simulation and impedance operator of a non local model of porous medium for acoustic control
}

\author{
Emmanuel Montseny*^, Céline Casenave ${ }^{\ddagger}$
}

\begin{abstract}
The problem under consideration relates to a model of a porous wall devoted to aircraft motor noise reduction. For such a medium, the parameters of the propagation equation depend on the frequency. Then, the associated time-model involves non rational convolution operators, which make the model complex from both analysis and simulation points of view.

In this paper, based on the so-called diffusive representation of convolution operators, a time-local formulation of the porous wall model, well adapted to analysis and numerical simulation, is established and analyzed. Then its associated impedance operator is computed. Finally, some numerical results relating to the time-domain simulation of the porous wall and its impedance operator are given.
\end{abstract}

\section{Introduction}

Aircraft motor noise reduction is currently an important challenge for aerospace industry. Control of acoustic perturbations can be active (Chatellier et al,

${ }^{*}$ CNRS, LAAS, 7 avenue du colonel Roche,F-31400 Toulouse, France.

${ }^{\dagger}$ Univ. de Toulouse, UPS, LAAS, F-31400, Toulouse, France.

${ }^{\ddagger}$ MODEMIC project-team, INRA/INRIA, UMR MISTEA, 2 place Pierre Viala, 34060 Montpellier, France.

Corresponding author:

Emmanuel Montseny, Laboratoire d'Analyse et d'Architecture des Systèmes, LAASCNRS, Université de Toulouse, 7 avenue du colonel Roche, 31077 Toulouse, France.

Email: emontseny@laas.fr 
2006; Levadoux and Montseny, 2003; Montseny, 2011) and/or passive, that is mainly based on specific materials, devoted to absorption of a wide part of the incident acoustic waves energy (Lai and Luo, 2008). In the case of hot zones such as exhaust nozzles, a porous wall made with welded hollow nickel balls was proposed in Gasser (2003). Porous materials are often used for the noise absorption thanks to their efficiency and their low cost. The modeling of the wave propagation inside these porous medium is nevertheless complex, especially because of the different possible structures of the medium. Moreover, the microscopic and macroscopic scales have both influence on the global behavior of the porous medium and have therefore to be both described in the model. Various models can be proposed depending on problem under consideration: prediction of the global behavior of the medium (Kidner and Hansen, 2008), understanding of some physical phenomena such as wave propagation, the fluid transport or the heat transfer in the medium (Amhalhel and Furmański, 1997), characterization of the structure or the physical characteristics of the medium (Vogel, 2002), etc. In this paper, the goal is to provide a model useful for noise control purposes. The global input-output behavior of the porous media has to be accurately described and summarized in such a way that the sound absorption efficiency of the porous wall can be estimated from numerical simulations. In that goal, an integro-differential model has been proposed in Gasser (2003) in the frequency domain. The interest of using such a type of models, compared to the other ones proposed in the literature (empirical models (Voronina, 1996), network models (Rege and Folger, 1987), fluid flows models (Layton et al, 2002), multiscale models (Biswal et al, 2009), etc), is that the macroscopic effects of the underlying microscopic phenomena are summarized and reduced, from a macroscopic point of view, by use of convolution operators. Thus, the complexity of the model is concentrated in convolution operators, on which the paper will focus. Such integro-differential models are often proposed to describe dissipative/dispersive materials; it is for example the case of the fractional diffusion equations (Mainardi et al, 2008; Caputo and Carcione, 2011). Note however that the fractional operators are not the only ones found in dissipative equations; the involved convolution operators can be more general, as it is the case in this paper.

The main difficulties encountered when manipulating a convolution operator $\mathcal{H}$ come from its time-non locality: the value of $(\mathcal{H} u)(t)$ depends on the whole history of $u$, that is on the restriction of $u$ to the past time interval ]$-\infty, t]$. On the contrary, an operator $\mathcal{H}: \mathfrak{X} \rightarrow \mathfrak{Y}$ is said to be time-local 
(or $t$-local) $)^{2}$ if for all $t \in \mathbb{R}$ and for any functions $u, v \in \mathfrak{X}$ equal on a neighborhood of $t$,

$$
(\mathcal{H} u)(t)=(\mathcal{H} v)(t) .
$$

The presence of time-non local operators makes the model more complex, from both analysis and simulation points of view. Indeed, standard techniques cannot be used because the involved dynamic operators are non rational, and the numerical complexity of the quadratures of convolution integrals generates highly expensive time discretizations, particularly when long memory components are present. In such situations, finite dimensional approximations of the convolution operators, like Padé ones (Baker and GravesMorris, 1996), can be used to get approximate differential models, easier to simulate and analyze. However, most of these constructions, which are mainly formal, do not ensure in practice the stability of the so-obtained models, and the physical relevance of the model parameters is often lost, which makes the analysis of the system tricky, or even impossible.

The results proposed in this paper ${ }^{3}$ are based on an original approach, devoted to integral causal operators, and called diffusive representation (Montseny, 2005; Casenave and Monsteny, 2010). This methodology allows to get timelocal input-output state realizations of convolution operators, well adapted to both analysis and numerical simulation. On the basis of this approach, we propose a formulation of the time-nonlocal operators (and therefore of the nonlocal model) in which only time-local operators are involved: such a formulation (or model) is said to be time-local ${ }^{4}$. The advantages of the time-locality are numerous. From the analysis point of view, some methods dedicated to differential (or partial differential) equations can be used: this is for example the case of energy methods which enables to prove the stability of a system or a numerical scheme and/or its dissipativity and passivity. Moreover, a time-local formulation often keeps a strong physical sense (contrary, for example, to Padé-based approximations). From the numerical point of view, the simulation of a time-local model is cheaper as it can be

\footnotetext{
${ }^{2}$ All differential operators are obviously time-local.

${ }^{3} \mathrm{~A}$ part of this work has already been presented in a conference paper at the IFAC World Congress 2008 (Casenave and Montseny (2008)) in a less detailed version, especially from the numerical point of view. The results of sections 3.1 and 3.2 are also given in a preliminary note (Casenave et al (2008)).

${ }^{4}$ The integral formulation $(\mathcal{H} u)(t)=\int_{0}^{t} h(t-s) u(s) d s$ is a classical time-nonlocal formulation of operator $\mathcal{H}$.
} 
solved by iterative methods ${ }^{5}$ : few storage and computations are therefore needed.

The paper is organized as follows. We introduce in section 2 the frequency model of the porous wall under consideration. In section 3, we build an equivalent time-local augmented input-output model of the medium. This new model is analyzed; in particular, we show that it is consistent from the point of view of the energy dissipation. Then, the coupling with a fluid medium is studied, and the global dissipativity is established. In section 4, we compute the analytic expression of the frequency response of the porous wall impedance operator. Then we propose a reduced diffusive formulation of this operator. Finally, some numerical results are presented in section 5 . The results of the diffusive representation theory required for this work are given in appendix A.

\section{Model under consideration}

In Gasser (2003), a porous material made with welded hollow nickel balls has been proposed, and a mono-dimensional homogenized model of this medium, based on its tubular structure and its physical properties, is considered. The frequency model under consideration describes the wave propagation inside the porous medium. It is given on $(\omega, z, x) \in \mathbb{R} \times] 0,1[\times] 0, X[$ by:

$$
\left\{\begin{array}{l}
e(x) \mathrm{i} \omega \rho_{\mathrm{eff}}(\mathrm{i} \omega) \hat{u}+\partial_{z} \hat{P}=0 \\
e(x) \mathrm{i} \omega \chi_{\mathrm{eff}}(\mathrm{i} \omega) \hat{P}+\partial_{z} \hat{u}=0
\end{array}\right.
$$

where $\widehat{u}$ and $\widehat{P}$ designate the Fourier transforms of the velocity and the pressure in the porous medium, $e(x)$ denotes the thickness of the porous wall and the parameters $\rho_{\text {eff }}(\mathrm{i} \omega)$ and $\chi_{\text {eff }}(\mathrm{i} \omega)$ are respectively the so-called effective density of Pride et al. (Pride et al, 1993) and the effective compressibility of Lafarge (Lafarge, 1993). These parameters are defined as follows:

$$
\left\{\begin{array}{l}
\rho_{\text {eff }}(\mathrm{i} \omega)=\rho\left(1+a \frac{\sqrt{1+b \mathrm{i} \omega}}{\mathrm{i} \omega}\right) \\
\chi_{\mathrm{eff}}(\mathrm{i} \omega)=\chi\left(1-c \frac{\mathrm{i} \omega}{\mathrm{i} \omega+a^{\prime} \sqrt{1+b^{\prime} \mathrm{i} \omega}}\right),
\end{array}\right.
$$

\footnotetext{
${ }^{5}$ The value of the solution at time $t$ can be obtained only from the value of the solution at time $t-\Delta t$ and the one of the input at time $t$.
} 
with:

$$
\begin{gathered}
\rho=\rho_{0} \alpha_{\infty}, \chi=\frac{1}{P_{0}}, a=\frac{8 \mu}{\rho_{0} \Lambda^{2}}, a^{\prime}=\frac{8 \mu}{\rho_{0} \Lambda^{\prime 2}}, \\
b=\frac{1}{2 a}, b^{\prime}=\frac{1}{2 a^{\prime}}, 0<c=\frac{\gamma-1}{\gamma}<1,
\end{gathered}
$$

where the physical parameters $\rho_{0}, P_{0}, \mu, \gamma, \alpha_{\infty}, \Lambda, \Lambda^{\prime}$ are respectively the density and the pressure at rest, the dynamic viscosity, the specific heat ratio, the tortuosity, the high frequency characteristic length of the viscous incompressible problem and the high frequency characteristic length of the thermal problem.

The model (1) is completed by the following boundary conditions:

$$
\left\{\begin{array}{l}
u_{\mid z=1}=0 \quad(\text { wave reflection at } z=1) \\
P_{\mid z=0}=w .
\end{array}\right.
$$

When coupling this system with the fluid medium, two interface conditions at the interface $\Gamma$ are necessary:

$$
\begin{gathered}
P_{\mid \Gamma}^{\text {fluid }}=P_{\mid z=0} \\
\text { and } u_{\mid \Gamma}^{\text {fluid }} \cdot n=\phi u_{\mid z=0},
\end{gathered}
$$

where $n$ is the outgoing unit normal on $\Gamma$ and $\phi>0$ is the porosity coefficient of the material. Thus, $w$ and

$$
y:=u_{\mid z=0}
$$

can be interpreted respectively as the input and the output of (1). The correspondence $w \mapsto y$ defines the impedance operator of the porous wall. This operator is expressed and studied in section 4 .

\section{State realization and analysis of the porous medium model}

Parameters $\rho_{\text {eff }}$ and $\chi_{\text {eff }}$ are non rational functions of the frequency $\omega$. As a consequence, the time model cannot be formulated as a finite dimensionnal state realization. Using the theory of diffusive representation, whose fundamental (and sufficient for this application) results are presented in appendix A, we give in the sequel a differential (an thus local) state realization in a suitable state-space. 


\subsection{Time-local formulation}

In this paragraph, we use the results of appendix A to transform the porous wall model (1). For that, we first express the time model obtained by inverse Fourier transform of (1); it is written:

$$
\left\{\begin{array}{l}
e(x)\left[\partial_{t} \circ \rho_{\mathrm{eff}}\left(\partial_{t}\right)\right] u+\partial_{z} P=0 \\
e(x)\left[\partial_{t} \circ \chi_{\mathrm{eff}}\left(\partial_{t}\right)\right] P+\partial_{z} u=0
\end{array}\right.
$$

where $\rho_{\text {eff }}\left(\partial_{t}\right)$ and $\chi_{\text {eff }}\left(\partial_{t}\right)$ are the (causal) convolution operators respectively associated to the symbols $\rho_{\text {eff }}$ and $\chi_{\text {eff }}$ (see formula $(2)$ ).

Consider the convolution operators $H_{1}\left(\partial_{t}\right)$ and $H_{2}\left(\partial_{t}\right)$ with respective symbols

$$
H_{1}(p)=\frac{1}{p \rho_{\mathrm{eff}}(p)} \text { and } H_{2}(p)=\frac{1}{p \chi_{\mathrm{eff}}(p)} .
$$

The system (1) can be written under the form:

$$
\left\{\begin{array}{l}
u=-\frac{1}{e} H_{1}\left(\partial_{t}\right) \partial_{z} P \\
P=-\frac{1}{e} H_{2}\left(\partial_{t}\right) \partial_{z} u
\end{array}\right.
$$

Proposition 1 The operators $-\frac{1}{e} \partial_{z} P \mapsto u$ and $-\frac{1}{e} \partial_{z} u \mapsto P$ involved in (7) can be realized by means of the following diffusive formulations:

$$
\left\{\begin{array} { l } 
{ \partial _ { t } \psi _ { 1 } = - \xi \psi _ { 1 } - \frac { 1 } { e } \partial _ { z } P } \\
{ u : = \langle \mu _ { 1 } , \psi _ { 1 } \rangle , }
\end{array} \quad \text { and } \left\{\begin{array}{l}
\partial_{t} \psi_{2}=-\xi \psi_{2}-\frac{1}{e} \partial_{z} u \\
P:=\left\langle\mu_{2}, \psi_{2}\right\rangle
\end{array}\right.\right.
$$

with $\mu_{1}$ and $\mu_{2}$ the respective $\gamma$-symbols of $H_{1}\left(\partial_{t}\right)$ and $H_{2}\left(\partial_{t}\right)$, which are given by:

$$
\begin{aligned}
& \mu_{1}(\xi)=\frac{a}{\pi \rho} \frac{\sqrt{b \xi-1}}{\xi^{2}+\frac{a \xi}{2}-a^{2}} \mathbf{1}_{\xi>2 a}+k_{1} \delta_{\xi_{1}}(\xi), \\
& \mu_{2}(\xi)=\frac{a^{\prime} c}{\pi \chi} \frac{\sqrt{b^{\prime} \xi-1}}{\xi^{2}(1-c)^{2}+\frac{a^{\prime}}{2} \xi-a^{\prime 2}} \mathbf{1}_{\xi>2 a^{\prime}}+\frac{1}{\chi} \delta_{0}(\xi)+k_{2} \delta_{\xi_{2}}(\xi),
\end{aligned}
$$

where:

$$
\begin{aligned}
& \xi_{1}=\frac{a(\sqrt{17}-1)}{4}>0, \xi_{2}=\frac{a^{\prime}\left(\sqrt{1+16(1-c)^{2}}-1\right)}{4(1-c)^{2}}>0, \\
& k_{1}=\frac{\sqrt{17}-1}{\rho \sqrt{17}}>0, \text { and } k_{2}=\frac{c\left(\sqrt{1+16(1-c)^{2}}-1\right)}{\chi(1-c) \sqrt{1+16(1-c)^{2}}}>0 .
\end{aligned}
$$


Proof. Functions $H_{1}$ and $H_{2}$ go to 0 at infinity and are analytic in $\mathbb{C} \backslash \mathbb{R}^{-}$. Let us determine their singularities ${ }^{6}$ :

- $H_{1}$ admits 2 singularities: a branch point in $-\frac{1}{b}$ and a simple pole in $a \frac{1-\sqrt{17}}{4}=-\xi_{1}$ which is the unique solution of $p+a \sqrt{1+b p}=0$.

- $H_{2}$ admits 3 singularities: a branch point in $-\frac{1}{b^{\prime}}$ and 2 simple poles in 0 and in $\frac{a^{\prime}\left(1-\sqrt{1+16(1-c)^{2}}\right)}{4(1-c)^{2}}=-\xi_{2}$, the last one being the unique solution of $p(1-c)+a^{\prime} \sqrt{1+b^{\prime} p}=0$.

As a consequence, $H_{1}\left(\partial_{t}\right)$ and $H_{2}\left(\partial_{t}\right)$ verify the hypotheses of corollary 4 (see appendix A). We so have, with $\psi$ solution of (38):

$$
\begin{aligned}
& \left(H_{1}\left(\partial_{t}\right) w\right)(t)=\left\langle\mu_{1}, \psi_{1}\right\rangle=\int_{\mathbb{R}^{+}} \mu_{1}^{c}(\xi) \psi(t, \xi) d \xi+\alpha_{1}^{1} \psi\left(t, \xi_{1}\right), \\
& \left(H_{2}\left(\partial_{t}\right) w\right)(t)=\left\langle\mu_{2}, \psi_{1}\right\rangle=\int_{\mathbb{R}^{+}} \mu_{2}^{c}(\xi) \psi(t, \xi) d \xi+\alpha_{0}^{2} \psi(t, 0)+\alpha_{1}^{2} \psi\left(t, \xi_{1}\right),
\end{aligned}
$$

with $^{7}$ :

$$
\begin{aligned}
\alpha_{1}^{1}= & \operatorname{Res}\left(H_{1},-\xi_{1}\right)=H_{1}(p)\left(p+\xi_{1}\right)_{\mid p=-\xi_{1}}=\frac{-\xi_{1}-a \sqrt{1-b \xi_{1}}}{\rho\left(-\xi_{1}-\frac{a(\sqrt{17}+1)}{4}\right)} \\
= & \frac{-2 \xi_{1}}{\rho\left(-\xi_{1}-\frac{a(\sqrt{17}+1)}{4}\right)}=\frac{\sqrt{17}-1}{\rho \sqrt{17}}, \\
\alpha_{0}^{2}= & \operatorname{Res}\left(H_{2}, 0\right)=\frac{1}{\chi_{\mathrm{eff}}(0)}=\frac{1}{\chi} \\
\alpha_{1}^{2}= & \operatorname{Res}\left(H_{2},-\xi_{2}\right)=H_{2}(p)\left(p+\xi_{2}\right)_{\mid p=-\xi_{1}} \\
= & \frac{\left(-\xi_{2}+a^{\prime} \sqrt{1-b^{\prime} \xi_{2}}\right)\left(-\xi_{2}(1-c)-a^{\prime} \sqrt{1-b^{\prime} \xi_{2}}\right)}{\chi\left(-\xi_{2}\right)(1-c)^{2}\left(-\xi_{2}-\frac{a^{\prime}}{4} \frac{\left.1+\sqrt{1+16(1-c)^{2}}\right)}{(1-c)^{2}}\right)} \\
= & \left.\frac{c\left(-\xi_{2}\right) 2(1-c)}{\chi(1-c)^{2}\left(-\xi_{2}-\frac{a^{\prime}}{4} \frac{1+\sqrt{1+16(1-c)^{2}}}{(1-c)^{2}}\right.}=\frac{\mathrm{i}(1-c) \sqrt{1+16(1-c)^{2}}}{\chi(1-)^{2}}, 1\right) \\
\text { and }= & \mu_{k}^{c}(\xi)=\frac{1}{2 i \pi} \lim _{n \rightarrow+\infty}\left[H_{k}\left(-\xi-\frac{1}{n}\right)-H_{k}\left(-\xi+\frac{\mathrm{i}}{n}\right)\right] \quad \xi \text {-ae. }
\end{aligned}
$$

\footnotetext{
${ }^{6}$ Note that the branch cut of the complex square root is here chosen equal to the negative real axis $\mathbb{R}^{-}$.

${ }^{7}$ For the computations, we use the relations $\xi_{1}=a \sqrt{1-\xi_{1} b}$ and $\xi_{2}(1-c)=a^{\prime} \sqrt{1-b^{\prime} \xi_{2}}$.
} 
By using the following result:

$$
\lim _{n \rightarrow+\infty} \sqrt{1+b\left(-\xi \pm \frac{\mathrm{i}}{n}\right)}=\left\{\begin{array}{c}
\sqrt{1-b \xi} \text { if } \xi \leqslant \frac{1}{b} \\
\pm \mathrm{i} \sqrt{b \xi-1} \text { if } \xi>\frac{1}{b}
\end{array}\right.
$$

we get the desired expressions of $\mu_{1}^{c}$ and $\mu_{2}^{c}$.

Then we deduce the following augmented model, defined on $(t, z, x, \xi) \in$ $\left.\mathbb{R}^{*+} \times\right] 0,1\left[\times \Gamma \times \mathbb{R}^{+}\right.$:

$$
\left\{\begin{array}{l}
\partial_{t} \psi_{1}=-\xi \psi_{1}-\frac{1}{e}\left\langle\mu_{2}, \partial_{z} \psi_{2}\right\rangle \\
\partial_{t} \psi_{2}=-\xi \psi_{2}-\frac{1}{e}\left\langle\mu_{1}, \partial_{z} \psi_{1}\right\rangle \\
\left\langle\mu_{1}, \psi_{1}(t, 1, x, .)\right\rangle=0 \\
\left\langle\mu_{2}, \psi_{2}(t, 0, x, .)\right\rangle=w(t, x) \\
y(t, x)=\left\langle\mu_{1}, \psi_{1}(t, 0, x, .)\right\rangle .
\end{array}\right.
$$

\subsection{Passivity of the model (8)}

Model (8) is time-local (equivalent to (1-3-5) from the input-output point of view), and is more convenient from both the analysis and the simulation point of view. Indeed, as $\mu_{1}$ and $\mu_{2}$ are positive, the functional

$$
\left(\psi_{1}, \psi_{2}\right) \mapsto\left\|\left(\psi_{1}, \psi_{2}\right)\right\|_{\mu, x}:=\left[\iint\left(\mu_{1}\left|\psi_{1}\right|^{2}+\mu_{2}\left|\psi_{2}\right|^{2}\right) d \xi d z\right]^{\frac{1}{2}}
$$

is a semi-norm, and we have:

Proposition 2 The functional $E_{\psi_{x}}=\frac{1}{2}\left\|\left(\psi_{1}, \psi_{2}\right)\right\|_{\mu, x}^{2}$ verifies on any solution $\left(\psi_{1}, \psi_{2}\right)$ of $(8)$ :

$$
\frac{d E_{\psi_{x}}}{d t}=-\int_{0}^{1} \int_{0}^{\infty} \xi \mu_{1}\left|\psi_{1}\right|^{2} d \xi d z-\int_{0}^{1} \int_{0}^{\infty} \xi \mu_{2}\left|\psi_{2}\right|^{2} d \xi d z+\frac{1}{e} w y .
$$

Proof.

$$
\begin{aligned}
\frac{d E_{\psi_{x}}}{d t}= & -\sum_{i} \int_{0}^{1} \int_{0}^{\infty} \xi \mu_{i}\left|\psi_{i}\right|^{2} d \xi d z-\frac{1}{e} \int_{0}^{1} P \partial_{z} u d z-\frac{1}{e} \int_{0}^{1} \partial_{z} P u d z \\
& =-\sum_{i} \int_{0}^{1} \int_{0}^{\infty} \xi \mu_{i}\left|\psi_{i}\right|^{2} d \xi d z-\frac{1}{e} P(t, 1, x) \underbrace{u(t, 1, x)}_{=0}+\frac{1}{e} P(t, 0, x) u(t, 0, x) .
\end{aligned}
$$


The two first terms of the right member of (9) are negative: they express the instantaneous dissipation of (8) for any given value of $x$, whereas the last term expresses the instantaneous exchanged power with the outside of the porous medium. Moreover, thanks to the property $\psi(t, 0)=0$ (which implies $E_{\psi_{x}}(0)=0$ ), and by integrating (9) from 0 to $T$, we deduce from (9) the positiveness of:

$$
w \mapsto \mathcal{Q}_{T}(w):=\int_{0}^{T} w y d t
$$

for any $T>0$ and any $x$. Thus, the passive feature of the absorbent wall is restored by model (8).

Note that (10) is a quadratic form, because, as we will show in the sequel, $y$ can be expressed as $y=Q\left(\partial_{t}\right) w$ with $Q\left(\partial_{t}\right)$ a linear dynamic operator.

\subsection{Coupling with an acoustic model}

As a consequence of (9), the coupling of (8) with another passive dynamic model leads to a globally dissipative system, provided that the energy functional is chosen such that the transfer between the two subsystems is balanced. This is shown in the sequel.

We consider classical acoustic model defined on a rectangular domain $] 0, Z[\times] 0, X[$, and couple this domain to a porous wall at the boundary $z=Z$; then, the whole model is written:

$$
\left\{\begin{array}{l}
\partial_{t}\left[\begin{array}{l}
u \\
\rho
\end{array}\right]+c_{0}\left[\begin{array}{cc}
0 & \nabla \\
\nabla^{T} & 0
\end{array}\right]\left[\begin{array}{l}
u \\
\rho
\end{array}\right]=f \\
u_{x}(t, z, 0)=u_{x}(t, z, X)=u_{z}(t, 0, x)=0 \\
u_{z}(t, Z, x)=\phi y(t, x) \\
w(t, x)=c_{0} \rho_{0} \rho(t, Z, x)
\end{array}\right.
$$

where $u$ and $\rho$ are the velocity and the density $\left(u_{x}\right.$ and $u_{z}$ are the velocities along the directions $x$ and $z$ respectively), $f$ is the perturbation source, $c_{0}$ is the velocity of wave fronts inside the fluid medium and $\phi$ is the porosity coefficient of the porous material. Note that the two last equations of (11) are the interface conditions (4). 
The acoustic energy of $(u, \rho)^{T}$ is classically given by the functional:

$$
E_{m}=\frac{1}{2}\left\|(u, \rho)^{T}\right\|_{L^{2}}^{2}
$$

Then, we can define a global energy for the coupled system (8-11) as:

$$
E=\int_{0}^{X} e(x) E_{\psi_{x}} d x+\frac{\rho_{0}}{\phi} E_{m}
$$

We have the following result:

Theorem 1 The system (8-11) is dissipative:

$\frac{d E}{d t}=-\int_{0}^{X} e(x) \int_{0}^{1} \int_{0}^{\infty} \xi \mu_{1}\left|\psi_{1}\right|^{2} d \xi d z d x-\int_{0}^{X} e(x) \int_{0}^{1} \int_{0}^{\infty} \xi \mu_{2}\left|\psi_{2}\right|^{2} d \xi d z d x \leqslant 0$ on any solution $\left(u, \rho, \psi_{1}, \psi_{2}\right)^{T}$ of (8-11).

Proof. We have:

$$
\begin{aligned}
\frac{d E_{m}}{d t} & =-\int_{0}^{X} \int_{0}^{Z} c_{0}\left[\begin{array}{cc}
0 & \nabla \\
\nabla^{T} & 0
\end{array}\right]\left[\begin{array}{l}
u \\
\rho
\end{array}\right] \cdot\left[\begin{array}{l}
u \\
\rho
\end{array}\right] d z d x \\
& =-c_{0} \int_{0}^{X} \int_{0}^{Z}\left(\partial_{x} \rho u_{x}+\partial_{z} \rho u_{z}+\partial_{x} u_{x} \rho+\partial_{z} u_{z} \rho\right) d z d x \\
& =-c_{0}\left(\int_{0}^{Z}\left[\rho u_{x}\right]_{x=0}^{X} d z+\int_{0}^{X}\left[\rho u_{z}\right]_{z=0}^{Z} d x\right) \\
& =-c_{0} \int_{0}^{X} \rho(t, Z, x) u_{z}(t, Z, x) d x=-\frac{\phi}{\rho_{0}} \int_{0}^{X} w(t, x) y(t, x) d x
\end{aligned}
$$

Then, by using (9) and after simple computations, we get:

$$
\frac{d E}{d t}=-\int_{0}^{X} e(x) \int_{0}^{1} \int_{0}^{\infty} \xi \mu_{1}\left|\psi_{1}\right|^{2} d \xi d z d x-\int_{0}^{X} e(x) \int_{0}^{1} \int_{0}^{\infty} \xi \mu_{2}\left|\psi_{2}\right|^{2} d \xi d z d x .
$$

Note that this global energy dissipation, due to wave absorption at the boundary, is not accessible under the original frequency or convolution forms (1) and (6). In that sense, the diffusive model (8) is in keeping with the physical interpretation of such media. 


\section{Impedance operator of the porous medium}

To build input-output state realizations of the porous medium model defined by (1-3-5), it can also be judicious to start from the symbol of the impedance operator $w \mapsto y$. This operator, denoted $Q\left(\partial_{t}\right)$ in the sequel, fully summarizes the porous wall action. Numerically, its realization is more accurate and cheaper than the simulation of the wave propagation inside the medium and so is more suitable, in particular for control purposes.

\subsection{Expression of the impedance operator}

In this section, the symbol of $Q\left(\partial_{t}\right)$ is computed. To simplify the notations, we do not write explicitly the dependence on $x$ of all the involved quantities.

Equations (1) of the porous medium can be written:

$$
\partial_{z} X=-e \mathrm{i} \omega A X
$$

with:

$$
X=\left[\begin{array}{c}
\widehat{P} \\
\hat{u}
\end{array}\right], A=\left[\begin{array}{cc}
0 & \rho_{\mathrm{eff}}(\mathrm{i} \omega) \\
\chi_{\mathrm{eff}}(\mathrm{i} \omega) & 0
\end{array}\right] .
$$

Diagonalization of $A$ leads to:

$$
A=M \underbrace{\left[\begin{array}{cc}
\sqrt{\chi_{\mathrm{eff}} \rho_{\mathrm{eff}}} & 0 \\
0 & -\sqrt{\chi_{\mathrm{eff}} \rho_{\mathrm{eff}}}
\end{array}\right]}_{D} M^{-1}
$$

where $M$ is the transition matrix. Using the change of unknowns $Y=M^{-1} X$, we get a diagonal system of ODEs:

$$
\partial_{z} Y=-e \mathrm{i} \omega D Y
$$

whose resolution leads to:

$$
\left\{\begin{array}{l}
Y_{1}(\omega, z)=Y_{1}(\omega, 0) \mathrm{e}^{-e \mathrm{i} \omega z \sqrt{\chi_{\text {eff }}(\mathrm{i} \omega) \rho_{\text {eff }}(\mathrm{i} \omega)}} \\
Y_{2}(\omega, z)=Y_{2}(\omega, 0) \mathrm{e}^{e \mathrm{i} \omega z \sqrt{\chi_{\text {eff }}(\mathrm{i} \omega) \rho_{\text {eff }}(\mathrm{i} \omega)}}
\end{array}\right.
$$

After simple computations, we deduce the following system on the original unknown $X=(\hat{P}, \hat{u})^{T}$ :

$$
\left\{\begin{array}{l}
\widehat{P}(\omega, z)=\frac{1}{2}\left(B+\frac{1}{B}\right) \widehat{P}(\omega, 0)+\frac{1}{2} \sqrt{\frac{\chi_{\mathrm{eff}}(\mathrm{i} \omega)}{\left.\rho_{\mathrm{eff}} \mathrm{i} \omega\right)}}\left(\frac{1}{B}-B\right) \widehat{u}(\omega, 0) \\
\hat{u}(\omega, z)=\frac{1}{2}\left(\frac{1}{B}-B\right) \widehat{P}(\omega, 0)+\frac{1}{2} \sqrt{\frac{\chi_{\mathrm{eff}}(\mathrm{i} \omega)}{\rho_{\mathrm{eff}}(\mathrm{i} \omega)}}\left(B+\frac{1}{B}\right) \widehat{u}(\omega, 0)
\end{array}\right.
$$


with:

$$
B(\omega, z):=\mathrm{e}^{\mathrm{i} \omega z \sqrt{\chi_{\text {eff }}(\mathrm{i} \omega) \rho_{\text {eff }}(\mathrm{i} \omega)} .}
$$

Using the boundary conditions (3) and expressions (13) with $z=1$, we get:

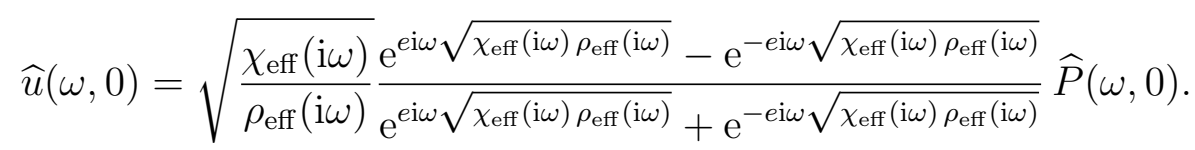

By denoting:

$$
A(\omega):=\mathrm{e}^{2 e \mathrm{i} \omega \sqrt{\chi_{\mathrm{eff}}(\mathrm{i} \omega) \rho_{\mathrm{eff}}(\mathrm{i} \omega)}}
$$

we finally have:

$$
\left\{\begin{array}{l}
\widehat{P}(\omega, z)=\frac{A(\omega) / B(\omega, z)+B(\omega, z)}{A(\omega)+1} \widehat{P}(\omega, 0) \\
\hat{u}(\omega, z)=\sqrt{\frac{\chi_{\mathrm{eff}}(\mathrm{i} \omega)}{\rho_{\mathrm{eff}}(\mathrm{i} \omega)}} \frac{A(\omega) / B(\omega, z)-B(\omega, z)}{A(\omega)+1} \widehat{P}(\omega, 0) .
\end{array}\right.
$$

The input-output correspondence $\widehat{w} \mapsto \widehat{y}=Q(\mathrm{i} \omega) \widehat{w}$ (which summarizes the frequency behavior of the porous medium) can then be deduced by taking $z=0$ in (16). Then, we finally get the following symbol of the porous medium impedance operator:

$$
Q(p)=\sqrt{\frac{\chi_{\mathrm{eff}}(p)}{\rho_{\mathrm{eff}}(p)}} \tanh \left(e(x) p \sqrt{\chi_{\mathrm{eff}}(p) \rho_{\mathrm{eff}}(p)}\right) .
$$

We show in the sequel that this operator admits an infinite dimensional state realization.

\subsection{Diffusive realization of the impedance operator}

First, we can note that:

$$
Q(p) \underset{\infty}{\sim} K_{1} \tanh \left(K_{2} p\right) \text { with } K_{1}=\sqrt{\frac{(1-c) \chi}{\rho}} \text { and } K_{2}=e(x) \sqrt{(1-c) \chi \rho}
$$

which implies that $Q(p) \underset{p \rightarrow \infty}{\longrightarrow} 1$. As a consequence, $Q\left(\partial_{t}\right)$ do not satisfy the hypotheses of theorem 2 (see appendix A). However, we can consider the operator $\widetilde{Q}\left(\partial_{t}\right)$ defined by the symbol $\widetilde{Q}(p):=\frac{Q(p)}{p}$, which is such that 
$\widetilde{Q}(p) \underset{p \rightarrow \infty}{\longrightarrow} 0$. Then, given a suitable contour $\gamma$, we know from (37) in appendix A that we can get the following state-representation of $Q\left(\partial_{t}\right)$ (from the realization of $\left.\widetilde{Q}\left(\partial_{t}\right)\right)$ :

$$
\left\{\begin{array}{l}
\partial_{t} \psi=\gamma \psi+w \\
y=\left\langle\nu, \partial_{t} \psi\right\rangle=\langle\gamma \nu, \psi\rangle+w\langle\nu, 1\rangle
\end{array}\right.
$$

where $\nu$ is the $\gamma$-symbol associated to $\widetilde{Q}\left(\partial_{t}\right)$ (note that, if the analytical expression of the $\gamma$-symbol $\nu$ is too complex to get, it is not necessarily needed for the numerical simulations ; see A.2.).

As regards the choice of a suitable contour $\gamma$, the operator $Q\left(\partial_{t}\right)$ (and then the operator $\widetilde{Q}\left(\partial_{t}\right)$ ) admits two kind of singularities: some branch points coming from the complex square roots, and an infinite sequence of simples poles (due to the presence of the function tanh in the symbol of the operator), as shown in Fig. 1. The impedance operator can therefore neither be realized with the same contour $\gamma$ as in section 3.1 nor with a sectoral contour of the form (44). A suitable contour $\gamma$, such that the operator $\widetilde{Q}\left(\partial_{t}\right)$ satisfies the hypotheses of theorem 2, can be built as shown on Fig. 2. However, this contour would not present all the interesting numerical properties of sectoral contours, which lead to cheap numerical realizations as mentioned in appendix $\mathrm{A}$.

For this reason, an approximation is finally made using a sectoral contour chosen such that a sufficient number of poles of $\widetilde{Q}(p)$ are surrounded by $\gamma$ (the other ones being in a way neglected). More precisely, given a sectoral contour $\gamma$, we consider the $\gamma$-diffusive operator with $\gamma$-symbol under the generic form (39), which is computed to be the best approximation of the operator $\widetilde{Q}$ in the sense of the least squares problem (43). Then, we deduce the approximate realization of $Q$ using (19). The influence of the sector's angle $\alpha$ on the quality of the approximation is illustrated in section 5 .

\section{$5 \quad$ Numerical results}

We give in this section some numerical results obtained by simulation of both the porous medium governed by (1-3) and the impedance operator $Q\left(\partial_{t}\right)$ of symbol (17). As described in appendix A.2, we can perform converging approximations of their respective diffusive realizations (8) and (19), by using 
a discretization $\left(\xi_{l}\right)_{l=1, L}$ of $\xi$ and standard quadratures. We then get approximate dynamic realizations of the form:

$$
\left\{\begin{array}{l}
\dot{X}=\mathcal{A} X+\mathcal{B} w \\
\widetilde{y}=\mathcal{C} X+\mathcal{D} w
\end{array} \quad \text { with } X(t) \in \mathbb{R}^{L},\right.
$$

such that $\widetilde{y} \simeq y$ in a suitable sense.

In this section, the numerical values of the involved physical parameters are (Gasser, 2003):

$$
\begin{aligned}
& \Lambda=\Lambda^{\prime}=0.110^{-3} \mathrm{~m}, \rho_{0}=1.2 \mathrm{~kg} \cdot \mathrm{m}^{-3}, P_{0}=10^{5} \mathrm{~Pa} \\
& \mu=1.810^{-5} \mathrm{~kg} \cdot \mathrm{m}^{-1} \cdot \mathrm{s}^{-1}, \gamma=1.4, \alpha_{\infty}=1.3, \quad e=210^{-2} \mathrm{~m} .
\end{aligned}
$$

\subsection{Simulations of the porous medium}

The explicit scheme used for the simulation of (8) is the one described and analyzed in Casenave and Montseny (2008). It is presented here-after.

Let consider the following time discretization:

$$
t_{0}=0, t_{n+1}=t_{n}+\Delta t, \forall n \geqslant 0
$$

with $\Delta t>0$ the time discretization step.

In model (8) only the thickness $e$ of the porous wall depends on the variable $x$. As a consequence, no discretization of the variable $x$ is necessary, the propagation of the waves for a given value of $x$ being independent from the others (tubular structure of the porous medium). Then, we consider $K$ spatial $z$-discretization points $\left\{z_{k}\right\}_{k=0: K}$ between 0 and 1 , which are defined by:

$$
z_{1}=0, z_{k+1}=z_{k}+\Delta z, \forall k=1: K-1,
$$

with $\Delta z>0$ the $z$-discretization step which is such that $\Delta z=\frac{1}{K-1}$.

As explained in section A.2 (appendix A), we also consider $L$ points of discretization $\xi_{l}^{L}, l=1: L$, of the variable $\xi$, geometrically spaced between $10^{2}$ and $10^{6}$ (i.e. such that the ratio $\frac{\xi_{l+1}^{L}}{\xi_{l}^{L}}$ is constant for all $l=1: L$ ):

$$
\xi_{1}^{L}=10^{2}, \xi_{l+1}^{L}=r \xi_{l}^{L}, \forall l=0: L-1,
$$

with $r>0$ the common ratio which is such that $\xi_{L}^{L}=r^{L} \xi_{0}^{L}=10^{6}$. 
Remark 1 The impact of the different discretizations (in $t, z$ and $\xi$ ) on the stability and precision of the numerical scheme is discussed in Casenave and Montseny (2008).

In the sequel, we denote $u^{n+1}(x), P^{n+1}(x)$ and $\psi_{i}^{n+1}\left(x, \xi_{l}^{L}\right), i=1: 2$ the vectors of $\mathbb{R}^{K}$ which are the approximations of:

$$
\begin{aligned}
u^{n+1}(x) & \simeq\left[u\left(t_{n+1}, x, z_{1}\right), \ldots, u\left(t_{n+1}, x, z_{K}\right)\right]^{T} \\
P^{n+1}(x) & \simeq\left[P\left(t_{n+1}, x, z_{1}\right), \ldots, P\left(t_{n+1}, x, z_{K}\right)\right]^{T} \\
\psi_{i}^{n+1}\left(x, \xi_{l}^{L}\right) & \simeq\left[\psi_{i}\left(t_{n+1}, x, z_{1}, \xi_{l}^{L}\right), \ldots, \psi_{i}\left(t_{n+1}, x, z_{K}, \xi_{l}^{L}\right)\right]^{T} .
\end{aligned}
$$

The used numerical scheme is based on the approximation of the derivative operator $\partial_{z}$ by centered finite differences, on the approximation of $\nu$ given by (39), and on the discretization of the $\psi$-equation given by (30). More details about this scheme can be found in Casenave and Montseny (2008). The scheme is written:

$$
\left\{\begin{array}{l}
\psi_{1}^{n+1}\left(x, \xi_{l}^{L}\right)=a_{l 1} \psi_{1}^{n-1}\left(x, \xi_{l}^{L}\right)-\frac{1}{e(x)} b_{l 1} G_{21} \sum_{j} b_{j 2} \psi_{2}^{n}\left(x, \xi_{j}^{L}\right) \\
\psi_{2}^{n+1}\left(x, \xi_{l}^{L}\right)=a_{l 2} \psi_{2}^{n-1}\left(x, \xi_{l}^{L}\right)-\frac{1}{e(x)} b_{l 2} G_{12} \sum_{j} b_{j 1} \psi_{1}^{n}\left(x, \xi_{j}^{L}\right) \\
u^{n+1}(x)=\sum_{l} b_{l 1} \psi_{1}^{n}\left(x, \xi_{l}^{L}\right) \\
P^{n+1}(x)=\sum_{l} b_{l 2} \psi_{2}^{n}\left(x, \xi_{l}^{L}\right)
\end{array}\right.
$$

with $a_{l 1}=a_{l 2}=e^{-2 \xi_{l}^{L} \Delta t}, b_{l i}=\sqrt{\mu_{l, i}^{L} \frac{1-e^{-2 \xi_{l}^{L} \Delta t}}{\xi_{l}}}$ where $\mu_{l, i}^{L}$ are the coefficients given by formula (41) for $\nu=\mu_{i}$, and

$$
G_{21}=G_{12}=\frac{1}{2 \Delta z}\left[\begin{array}{ccccc}
0 & 1 & & & \\
-1 & 0 & 1 & & \\
& \ddots & \ddots & \ddots & \\
& & -1 & 0 & 1 \\
& & & -1 & 0
\end{array}\right] \text {. }
$$

Remark 2 Because $\mu_{1}$ and $\mu_{2}$ are positive measures, the coefficients $\mu_{l, i}^{L}$ are also positive.

The boundary conditions we used are:

$$
\begin{aligned}
u(t, 1) & =0, \\
P(t, 0) & =w(t)=\left(1-\cos \left(2 \pi \times 1.310^{4} t\right)\right) \mathbf{1}_{[0, T]}(t) .
\end{aligned}
$$


The frequency responses of the approximations of $H_{1}\left(\partial_{t}\right)$ and $H_{2}\left(\partial_{t}\right)$, respectively obtained with $L=15$ and $L=20$, are given in figures 3 and 4 . Recall that the $\xi$-discretization covers 4 decades, from $10^{2}$ to $10^{6}$. We can see that the approximate frequency responses fit the analytic ones on the desired bandwidth. 


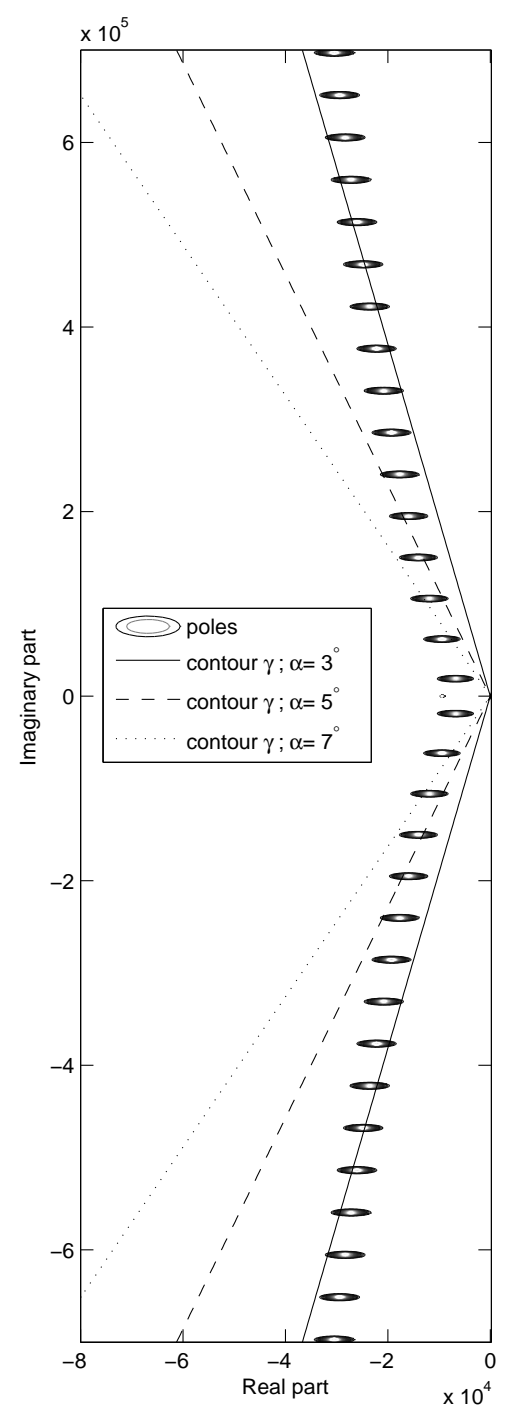

Figure 1: Poles of $Q(p)$ 


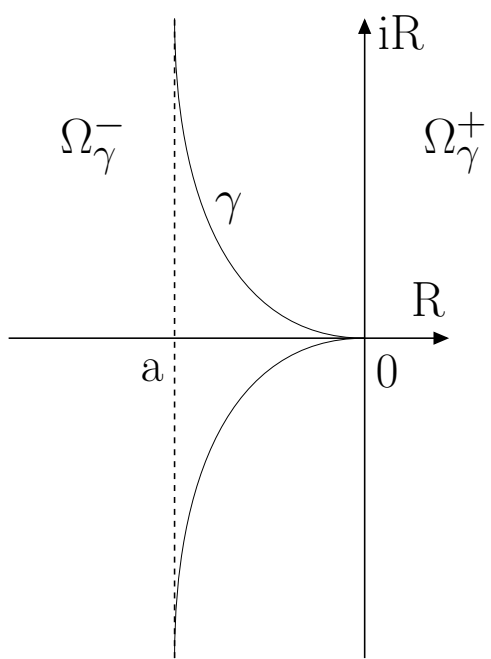

Figure 2: Example of contour $\gamma$ suitable for the state realization of $\tilde{Q}\left(\partial_{t}\right)$ 

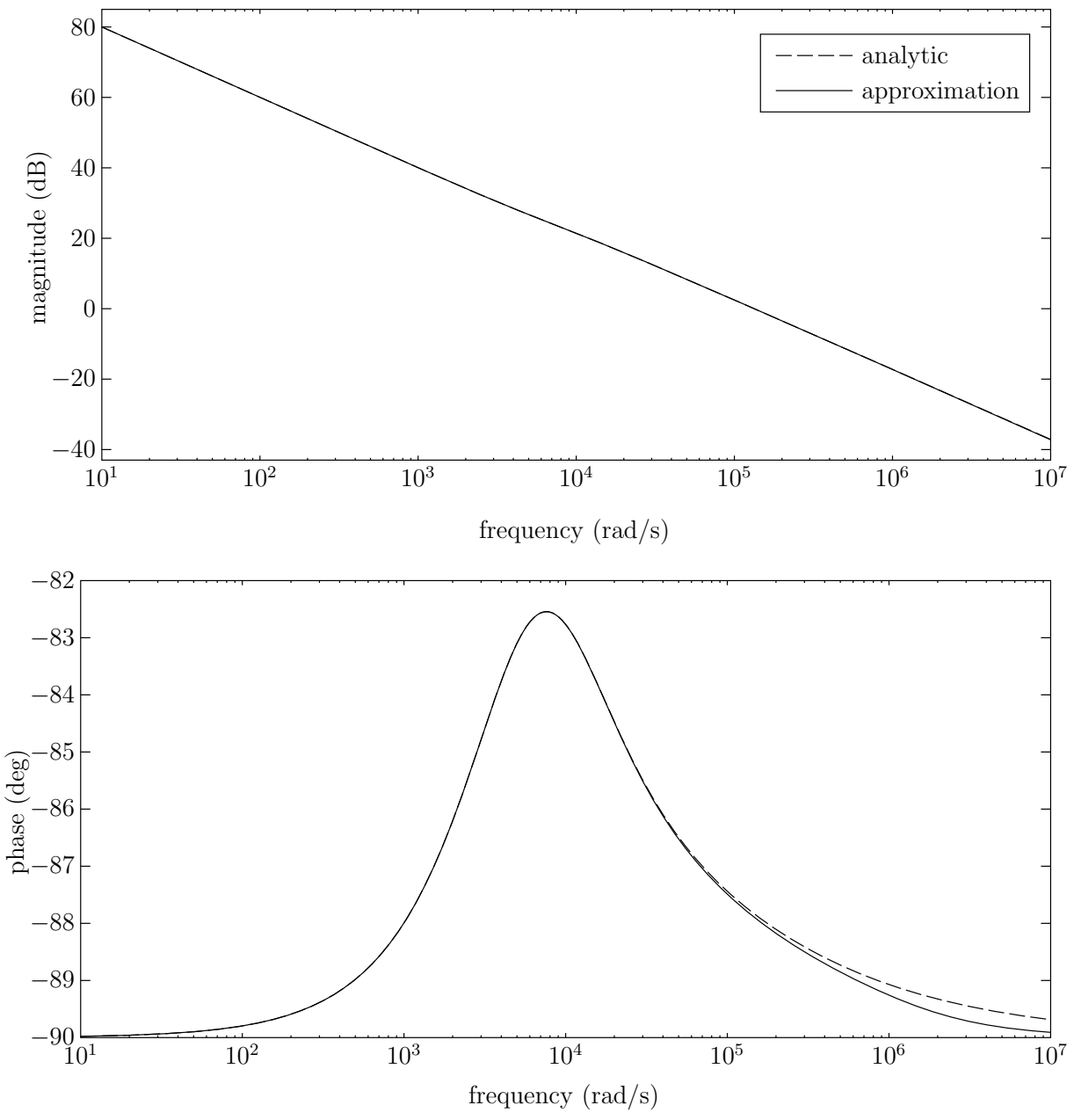

Figure 3: Frequency response of operator $H_{1}\left(\partial_{t}\right)$ 

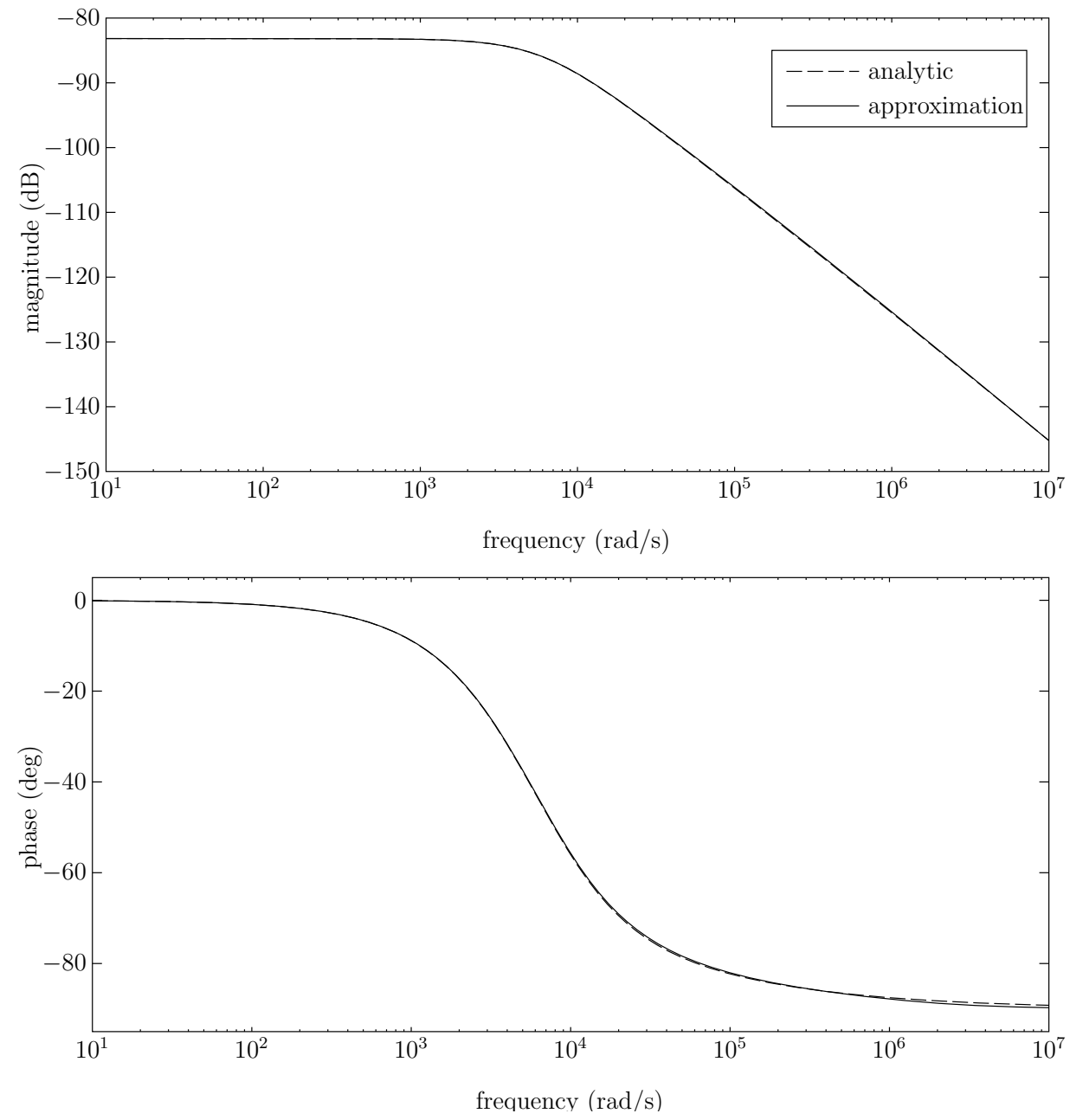

Figure 4: Frequency response of operator $H_{2}\left(\partial_{t}\right)$ 
We give in figure 5 the profile of $P$ in the porous medium at different times $t$. We clearly observe the dissipative and dispersive nature of this propagation model, due to the convolution operators $H_{1}\left(\partial_{t}\right)$ and $H_{2}\left(\partial_{t}\right)$. As an illustration, we give in figure 6 the functions $\psi_{1}$ involved in the synthesis of $u$, at a fixed time $t=0.09 \mathrm{~ms}$. 

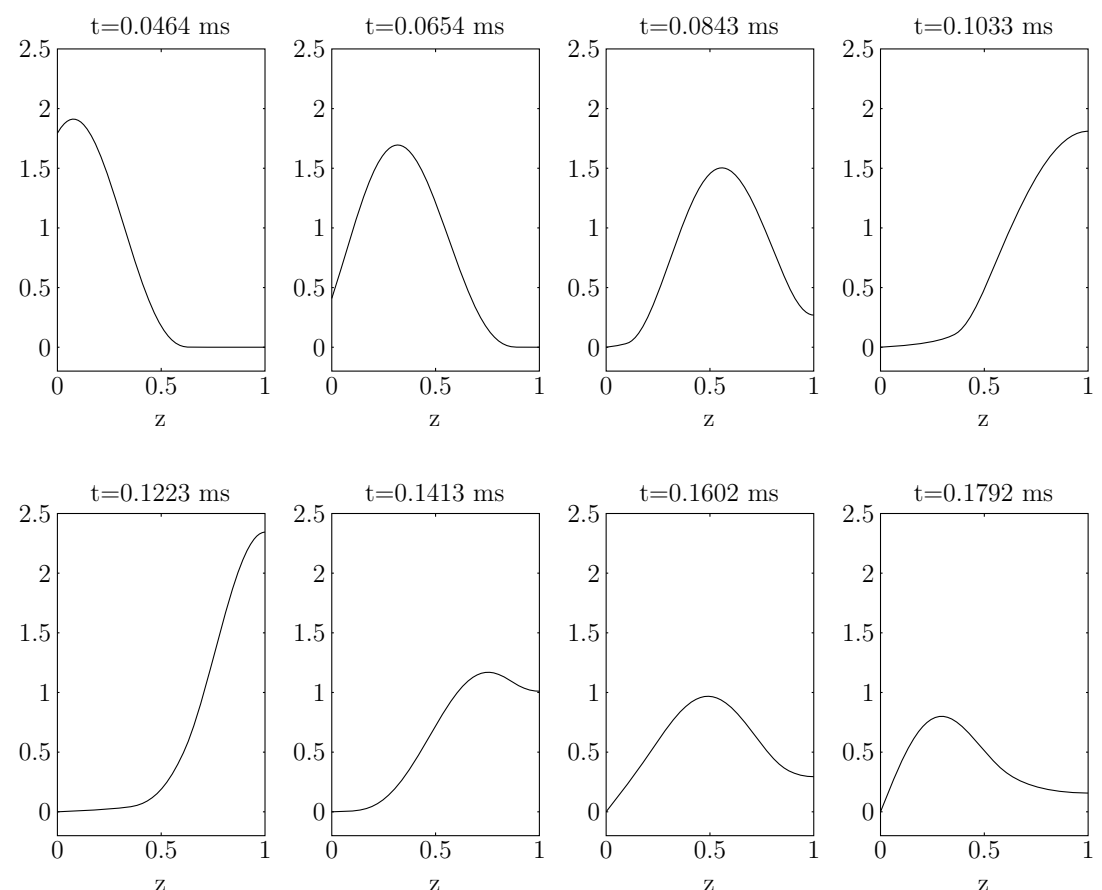

Figure 5: Evolution of $P=\sum_{l} b_{l 2} \psi_{2}\left(\xi_{l}\right)$ (N.B: the unit of length for the $z$-axis is $e=2.10^{-2} \mathrm{~m}$ )

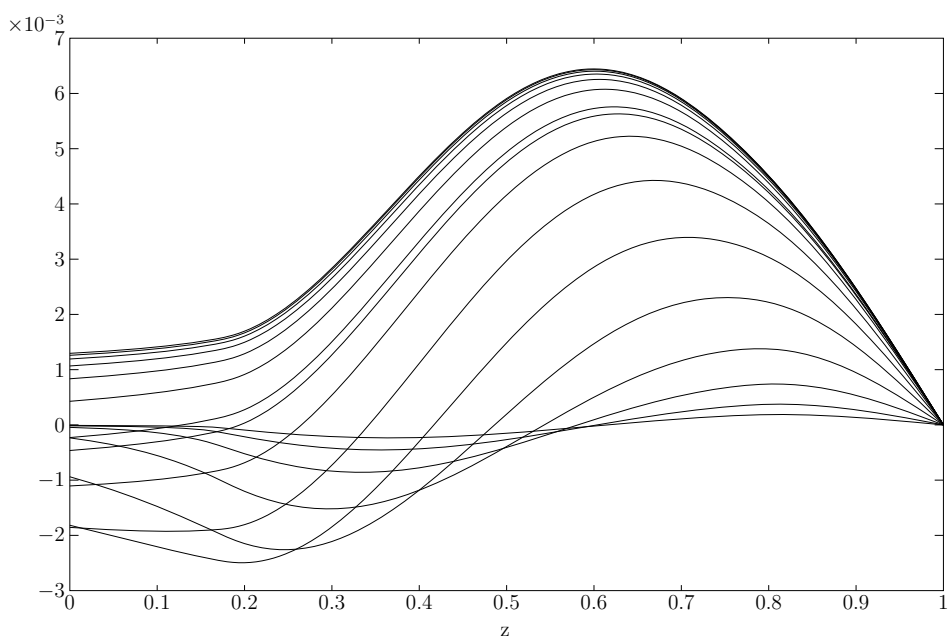

Figure 6: Functions $\psi_{1}\left(t, ., x, \xi_{l}\right) l=1: L$ at time $t=0.09 \mathrm{~ms}$. 
Note that even if the bode diagrams of $H_{1}\left(\partial_{t}\right)$ and $H_{2}\left(\partial_{t}\right)$ seem to be rather "basic", and in spite of the small value of parameters $b$ and $b^{\prime}(\simeq$ $\left.4.210^{-5}\right)$ in $\chi_{\text {eff }}$ and $\rho_{\text {eff }}$, these operators cannot be well approximated by first-order operators by considering $b=b^{\prime}=0$, as highlighted in figure 9 . The integro-differential nature of operators $\chi_{\text {eff }}$ and $\rho_{\text {eff }}$ is essential to simulate the porous wall behavior accurately.

\subsection{Simulations of the impedance operator}

In this paragraph, we give some indications and numerical results about the simulation of (19).

To simulate (19), we first consider the finite dimensional approximate state formulation of $Q\left(\partial_{t}\right)$ obtained from (40), (36) and (19), and given by:

$$
\left\{\begin{array}{l}
\partial_{t} \psi\left(t, \xi_{l}^{L}\right)=\gamma\left(\xi_{l}^{L}\right) \psi\left(t, \xi_{l}^{L}\right)+w(t), l=1: L \\
\psi\left(0, \xi_{l}^{L}\right)=0, l=1: L \\
y(t):=\left[Q\left(\partial_{t}\right) w\right](t) \simeq 2 \operatorname{Re}\left(\sum_{l=1}^{L} \nu_{l}^{L} \gamma\left(\xi_{l}^{L}\right) \psi\left(t, \xi_{l}^{L}\right)+w(t) \sum_{l=1}^{L} \nu_{l}^{L}\right)
\end{array}\right.
$$

The discretization points $\xi_{l}^{L}$ covers 4 decades from $10^{2}$ to $10^{6}$ with $L=250$ and are geometrically spaced. The coefficients $\nu_{l}^{L}$ have been obtained by resolution of the least squares problem (43) with $H(p)=\tilde{Q}(p)$.

The analytical solution $\psi\left(t, \xi_{l}^{L}\right)$ of the state equation is given by:

$$
\psi\left(t, \xi_{l}^{L}\right)=\int_{0}^{t} e^{\gamma\left(\xi_{l}^{L}\right)(t-s)} w(s) d s .
$$

Assume that the function $w$ is constant on the time interval $[t, t+\Delta t]$. We then get, after computations:

$$
\psi\left(t+\Delta t, \xi_{l}^{L}\right)=e^{\gamma\left(\xi_{l}^{L}\right) \Delta t} \psi\left(t, \xi_{l}^{L}\right)+\frac{e^{\gamma\left(\xi_{l}^{L}\right) \Delta t}-1}{\gamma\left(\xi_{l}^{L}\right)} w(t) .
$$

This numerical scheme is the one which has been used for the simulation of the impedance operator $\tilde{Q}\left(\partial_{t}\right)$ : its stability comes from the fact that $\left|e^{\gamma\left(\xi_{l}^{L}\right) \Delta t}\right|=e^{\operatorname{Re}\left(\gamma\left(\xi_{l}^{L}\right)\right) \Delta t} \leqslant 1$ because $\operatorname{Re}\left(\gamma\left(\xi_{l}^{L}\right)\right) \leqslant 0$.

Several approximations of the impedance operator, obtained by using different sectoral contours $\gamma$ (of the form (44)) with respective angle $\alpha=3^{\circ}$, $5^{\circ}$ and $7^{\circ}$ (see figure 1) have been compared. The frequency responses of the so-obtained approximations of $\widetilde{Q}\left(\partial_{t}\right)$ are given in figure 7 . 

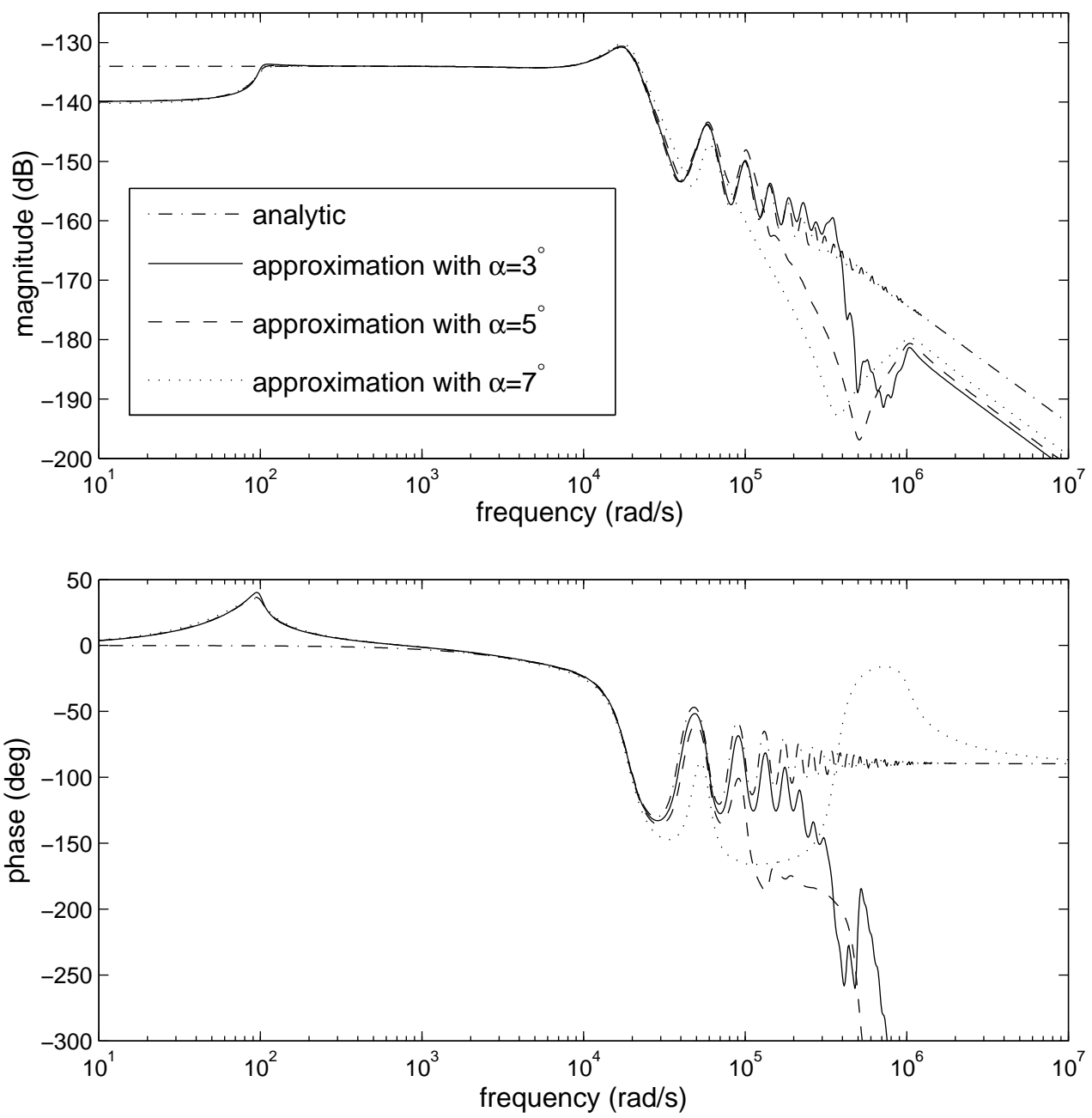

Figure 7: Frequency response of operator $\widetilde{Q}\left(\partial_{t}\right)$ for different values of the sector angle $\alpha$. 
In figure 8 , the evolution of the output $y=u_{\mid z=0}$ obtained with the 3 different approximations of $\widetilde{Q}\left(\partial_{t}\right)$ is plotted, with input $w$ given by (28). As said previously, the larger the number of poles included by $\gamma$, the better the approximation of the impedance operator.

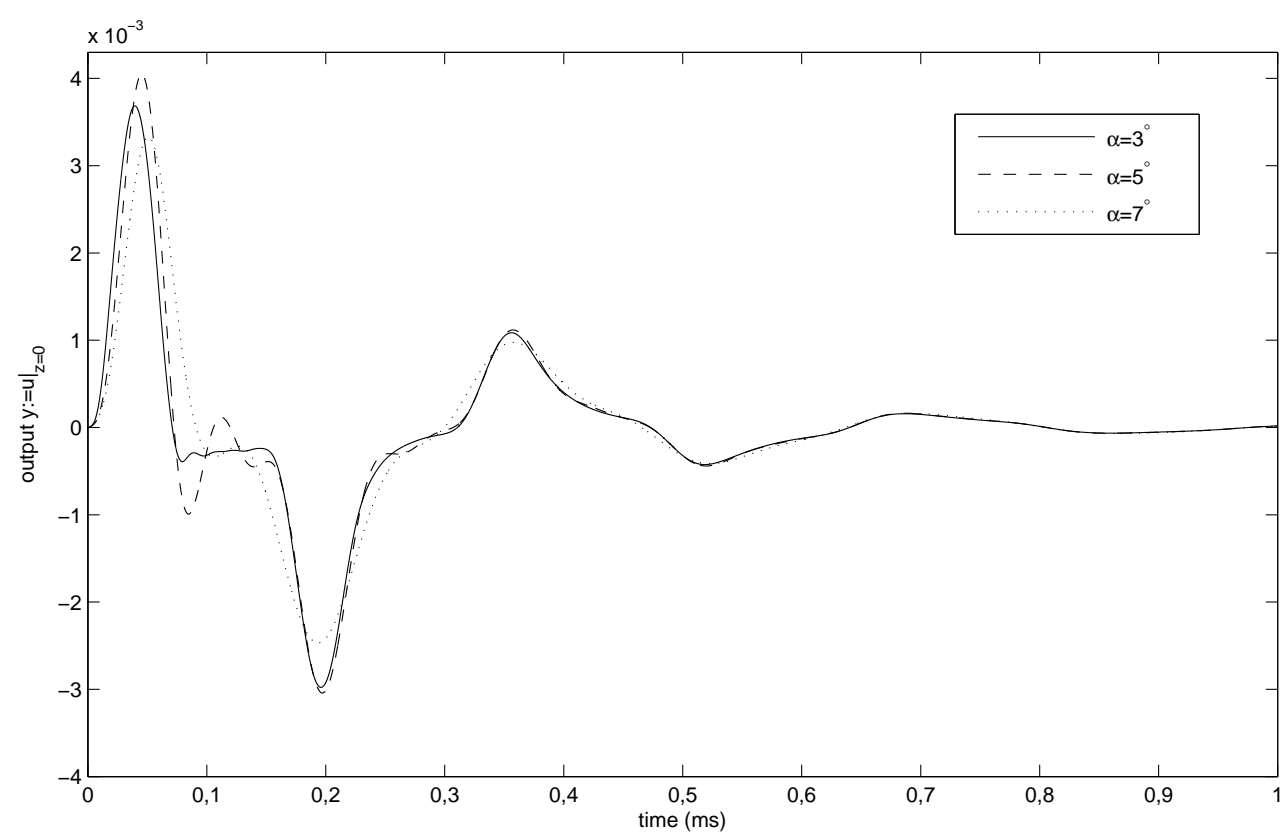

Figure 8: Influence of the angle $\alpha$ on the quality of the impedance operator approximation.

Finally, figure 9 shows the output $y:=u_{\mid z=0}$ obtained by simulation of the impedance operator (with $\alpha=3^{\circ}$ ) with $w$ given by (28), compared with the value of $u_{\mid z=0}$ obtained by simulating the whole porous medium, as described in previous paragraphs. As expected, the results are quite similar; however, as the simulation of (19) does not need any $z$-discretization, simulation of (19) is cheaper than the scheme (26). 


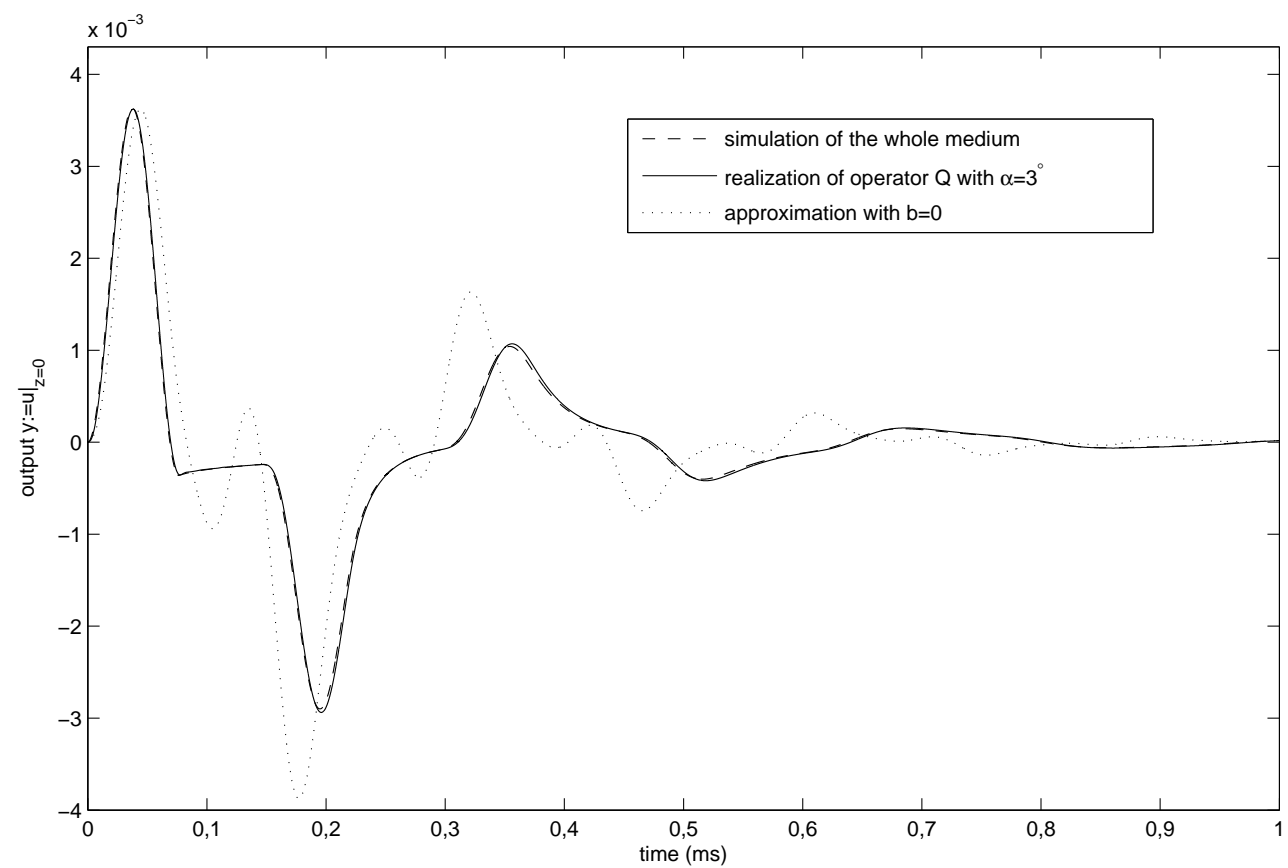

Figure 9: Comparison of the evolution of the output $y:=\left.u\right|_{z=0}$ obtained by simulation of the whole medium with the one obtained by realization of the impedance operator.

\section{Conclusion}

In this paper, we propose a new formulation of an integro-differential model of porous media. From this formulation, the dissipative and passive features of the porous wall are easily established, and simple and precise numerical simulations of the model are given. Thanks to those results, the simulation of the coupling between a fluid medium and the porous wall can be planned in future works.

The porous wall impedance operator is also computed, and realized with an approximate diffusive formulation. This operator has the advantage to summarize the boundary behavior of the porous wall, which makes its implementation cheaper and more precise than the whole simulation of the porous wall model, especially for active control purposes. 


\section{A Diffusive realizations of causal convolution operators}

In this section, we present a particular case of a methodology called "Diffusive representation", introduced and developed by G. Montseny in a general framework (Montseny, 2005; Casenave and Monsteny, 2010). The diffusive representation theory allows, under few hypotheses, to get state representations of convolution operators. Various applications and questions relating to this approach can be found in many papers (Audounet et al, 1998; Carmona and Coutin, 1998; Degerli et al, 1999; Garcia and Bernussou, 1998; Levadoux and Michielsen, 1998; Montseny, 2007; Mouyon and Imbert, 2002; Rumeau et al, 2006).

\section{A.1 Mathematical framework}

Consider a causal convolution operator defined, on any continuous function $w: \mathbb{R}^{+} \rightarrow \mathbb{R}$, by:

$$
w \mapsto\left(t \mapsto \int_{0}^{t} h(t-s) w(s) d s=(h * w)(t)\right) .
$$

Let denote $H$ the Laplace transform of $h$ and $H\left(\partial_{t}\right)$ the convolution operator defined by (31). The function $H(p)$ is often called the (Laplace-)symbol of the operator $H\left(\partial_{t}\right)$.

Let $W_{\text {loc }}^{1, \infty}(\mathbb{R} ; \mathbb{C})$ denote the topological space of measurable functions $f: \mathbb{R} \rightarrow \mathbb{C}$ such that $f, f^{\prime} \in L_{\text {loc }}^{\infty}$ (that is $f$ and $f^{\prime}$ are locally bounded in the "almost everywhere" sense). The convergence $f_{n} \rightarrow f$ in $W_{\text {loc }}^{1, \infty}$ means that, on any bounded set $P, f_{n \mid P}-f_{\mid P} \rightarrow 0$ and $f_{n \mid P}^{\prime}-f_{\mid P}^{\prime} \rightarrow 0$ uniformly.

Let $\gamma$ be a closed (possibly at infinity) simple arc in $\mathbb{C}^{-}$, defined by a function of $W_{\text {loc }}^{1, \infty}(\mathbb{R} ; \mathbb{C})$, also denoted $\gamma$, such that $\gamma(0)=0$. We denote $\Omega_{\gamma}^{+}$ the exterior domain defined by $\gamma$, and $\Omega_{\gamma}^{-}$the complementary of $\overline{\Omega_{\gamma}^{+}}$(see figure 10). Then, we have the following theorem (Montseny, 2005; Casenave and Monsteny, 2010).

Theorem 2 If $H$ is holomorphic in $\Omega_{\gamma}^{+}$, if $H(p) \rightarrow 0$ when $|p| \rightarrow \infty$ in $\Omega_{\gamma}^{+}$, and if the possible singularities of $H$ on $\gamma$ are simple poles or branch points such that ${ }^{8}|H \circ \gamma|$ is locally integrable in their neighborhood, then with:

\footnotetext{
${ }^{8}$ The symbol $\circ$ stands for the composition of functions: $H \circ \gamma$ is therefore the function $\xi \mapsto H(\gamma(\xi))$.
} 


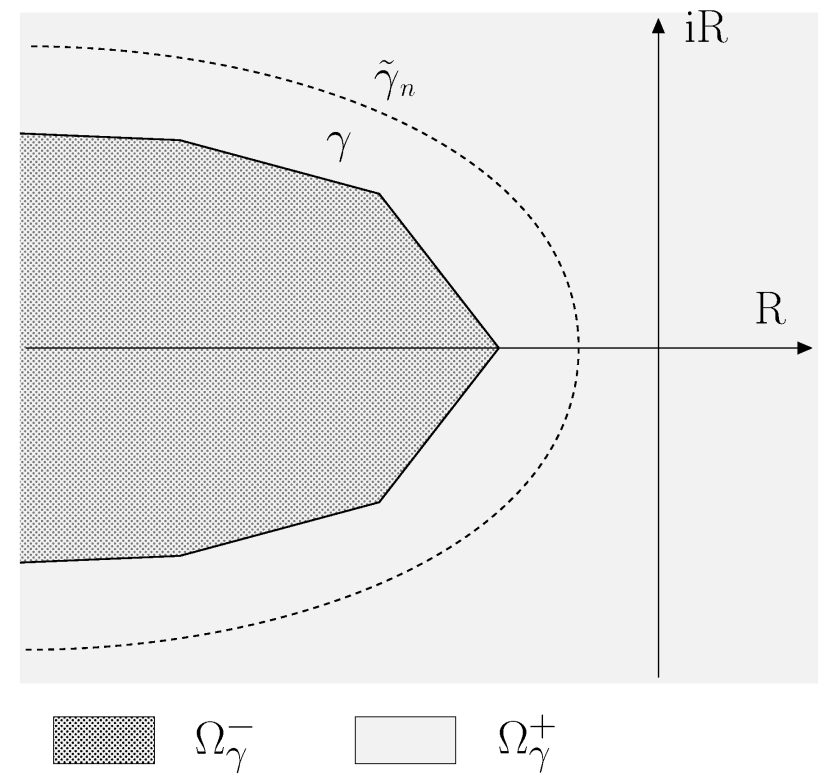

Figure 10: Example of $\gamma$ and $\widetilde{\gamma}_{n}$ arcs.

- $\tilde{\gamma}_{n}$ a sequence of closed simple arcs in $\Omega_{\gamma}^{+}$(defined by some functions of $W_{\text {loc }}^{1, \infty}(\mathbb{R} ; \mathbb{C})$ also denoted $\left.\tilde{\gamma}_{n}\right)$, such that, for any $n, \gamma \subset \Omega_{\tilde{\gamma}_{n}}^{-}$and:

$$
\tilde{\gamma}_{n} \underset{n \rightarrow \infty}{\longrightarrow} \gamma \text { in } W_{\text {loc }}^{1, \infty}, \quad \text { (see figure 10) }
$$

- $\nu$ the measure defined by:

$$
\nu=\frac{\gamma^{\prime}}{2 \mathrm{i} \pi} \lim _{n \rightarrow \infty} H \circ \tilde{\gamma}_{n} \text { in the sense of measures, }
$$

we have ${ }^{9}$ :

$$
\left[H\left(\partial_{t}\right) w\right](t)=\langle\nu, \psi(t, .)\rangle_{\xi \in \mathbb{R}},
$$

where $\psi(t, \xi)$ is solution of the following evolution problem on $(t, \xi) \in \mathbb{R}^{*+} \times \mathbb{R}$ :

$$
\partial_{t} \psi(t, \xi)=\gamma(\xi) \psi(t, \xi)+w(t), \psi(0, \xi)=0 .
$$

\footnotetext{
${ }^{9}$ We often indifferently denote $\langle\nu, \psi\rangle$ or $\int \nu \psi d \xi$ the duality product between a measure $\nu$ and a continuous function $\psi$. In particular, when $\nu$ is atomic, i.e. $\nu=\sum_{k} a_{k} \delta_{\xi_{k}}$, we have: $\langle\nu, \psi\rangle=\sum_{k} a_{k} \psi\left(\xi_{k}\right)$.
} 
Definition 3 - The measure $\nu$ defined by (33) is called the canonical $\gamma$-symbol of the operator $H\left(\partial_{t}\right)$.

Any measure $\bar{\nu}:=\nu+\nu_{0}$ with $\nu_{0}$ such that $\left\langle\nu_{0}, \psi(t,).\right\rangle=0, \forall \psi$, is also called a $\gamma$-symbol of $H\left(\partial_{t}\right)$.

- The function $\psi$ solution of (35) is called the $\gamma$-representation of $w$.

Remark 3 Note that, thanks to (34), the Dirac measure $\delta$ is clearly a $\gamma$ symbol of the operator $w \mapsto \int_{0}^{t} w(s) d s$, denoted $\partial_{t}^{-1}$. We indeed have $\left(\partial_{t}^{-1} w\right)(t)=$ $\langle\delta, \psi(t,)\rangle=.\psi(t, 0)$, with $\partial_{t} \psi(t, 0)=w, \psi(0,0)=0$.

Remark 4 When the functions $h=\mathcal{L}^{-1}(H)$ and $w$ take only real values, and when $\gamma$ is symmetric with respect to the real axis, that is $\gamma(\xi)=\overline{\gamma(-\xi)}, \forall \xi$, then, it can be shown (Montseny (2005)):

$$
\left[H\left(\partial_{t}\right) w\right](t)=\langle\nu, \psi(t, .)\rangle_{\xi \in \mathbb{R}}=2 \operatorname{Re}\left(\langle\nu, \psi(t, .)\rangle_{\xi \in \mathbb{R}^{+}}\right) .
$$

Remark 5 Formulation (35,34) can easily be extended to operators of the form $H\left(\partial_{t}\right):=Q\left(\partial_{t}\right) \circ \partial_{t}$ where $Q\left(\partial_{t}\right)$ admits a $\gamma$-symbol. From (35), we then have formally:

$$
\left[H\left(\partial_{t}\right) w\right](t)=\left\langle\nu, \partial_{t} \psi_{w}(t, .)\right\rangle=\left\langle\nu, \gamma \psi_{w}(t, .)+w(t)\right\rangle
$$

with $\nu$ the $\gamma$-symbol of $Q\left(\partial_{t}\right)=H\left(\partial_{t}\right) \circ \partial_{t}^{-1}$.

In spite of its apparent complexity, the use of Theorem 2 is rather simple: if we can find an $\operatorname{arc} \gamma$ which surrounds (in the complex plane) all the singularities of the Laplace-symbol $H(p)$, and which is such that $H(p) \rightarrow 0$ when $|p| \rightarrow \infty$ in $\Omega_{\gamma}^{+}$, then we can realize the operator $H\left(\partial_{t}\right)$ with the model $(35,34)$, which is composed of a time-local (differential) state equation of infinite dimension, and of a dual product (generalized integral).

In the case where $\gamma(\xi)=-|\xi|$, the result can be simplified; we get:

Corollary 4 If $H$ is analytic on $\mathbb{C} \backslash \mathbb{R}^{-}$with a discrete set of singularities in $\mathbb{R}^{-}$, if in addition these singularities are either simple poles $-\xi_{k}$ or branch points such that $|H|$ is locally integrable, and if $H(p) \rightarrow 0$ when $p \rightarrow \infty$, then:

$$
\left(H\left(\partial_{t}\right) w\right)(t)=\int_{0}^{+\infty} \mu^{c}(\xi) \psi(t, \xi) d \xi+\sum_{k} \alpha_{k} \psi\left(t, \xi_{k}\right)=\langle\mu, \psi(t, .)\rangle_{\mathbb{R}^{+}}
$$


where ${ }^{10}$ :

$$
\begin{aligned}
\mu^{c} \in L_{\mathrm{loc}}^{1}\left(\mathbb{R}^{+}\right), \quad \mu^{c}(\xi)= & \frac{1}{2 i \pi} \lim _{n \rightarrow+\infty}\left[H\left(-\xi-\frac{\mathrm{i}}{n}\right)-H\left(-\xi+\frac{\mathrm{i}}{n}\right)\right] \quad \xi-a e, \\
\alpha_{k}=\operatorname{Res}\left(H,-\xi_{k}\right), & \mu=\mu^{c}+\sum_{k} \alpha_{k} \delta_{\xi_{k}},
\end{aligned}
$$

and $\psi(t, \xi)$ is the unique solution of the following Cauchy problem on $(t, \xi) \in$ $\mathbb{R}^{*+} \times \mathbb{R}^{+}$

$$
\partial_{t} \psi(t, \xi)=-\xi \psi(t, \xi)+w(t), \psi(0, \xi)=0 .
$$

Proof. (sketch)

From symmetry of the problem $\left(\gamma(\xi)=\gamma(-\xi), \gamma^{\prime}(\xi)=-\gamma^{\prime}(-\xi)\right.$ and $\psi(t, \xi)=$ $\psi(t,-\xi))$, we have:

$$
\left\langle\frac{\gamma^{\prime}}{2 \mathrm{i} \pi} H \circ \tilde{\gamma}_{n}, \psi(t, .)\right\rangle_{\mathbb{R}}=\left\langle\frac{\gamma^{\prime}(-.)}{2 \mathrm{i} \pi}\left(H \circ \tilde{\gamma}_{n}(-.)-H \circ \tilde{\gamma}_{n}\right), \psi(t, .)\right\rangle_{\mathbb{R}^{+}} .
$$

Consider the $\operatorname{arcs} \widetilde{\gamma}_{n}$ given in figure 11. We have:

- for all $\xi \in \mathbb{R}^{+}$such that $-\xi$ isn't a singularity of $H$, there exists $n^{*} \in \mathbb{N}$ such that, for all $n \geqslant n^{*}$ :

$$
\widetilde{\gamma}_{n}(\xi)=-\xi+\frac{\mathrm{i}}{n} \text { and } \widetilde{\gamma}_{n}(-\xi)=-\xi-\frac{\mathrm{i}}{n} .
$$

As $\gamma^{\prime}(-\xi)=1$, we get the expression of $\mu^{c}$.

- for all simple poles $-\xi_{k}$, we have:

$$
\left\langle\frac{\gamma^{\prime}(-.)}{2 \mathrm{i} \pi}\left(H \circ \tilde{\gamma}_{n}(-.)-H \circ \tilde{\gamma}_{n}\right), \psi(t, .)\right\rangle_{\left[-\xi_{k}-\frac{1}{n}, \xi_{k}+\frac{1}{n}\right]}=\frac{1}{2 \mathrm{i} \pi} \int_{c_{n}^{k}} H(p) \Psi(t, p) d p,
$$

where $c_{n}^{k}$ is the contour which follows the circle of center $-\xi_{k}$ and radius $\frac{1}{n}$ in the counter-clockwise sense, and $\Psi(t,$.$) is the analytic continuation$ of $\psi(t,$.$) . As \Psi(t,$.$) is analytic on \mathbb{C}$, and $\psi(t, \xi)=\Psi(t,-\xi)$ we have:

$$
\frac{1}{2 \mathrm{i} \pi} \int_{c_{n}^{k}} H(p) \Psi(t, p) d p=\operatorname{Res}\left(H \Psi(t, .),-\xi_{k}\right)=\operatorname{Res}\left(H,-\xi_{k}\right) \psi\left(t, \xi_{k}\right) .
$$

\footnotetext{
${ }^{10} \operatorname{Res}(f, a)$ is the residue of the function $f$ at the point $a$.
} 


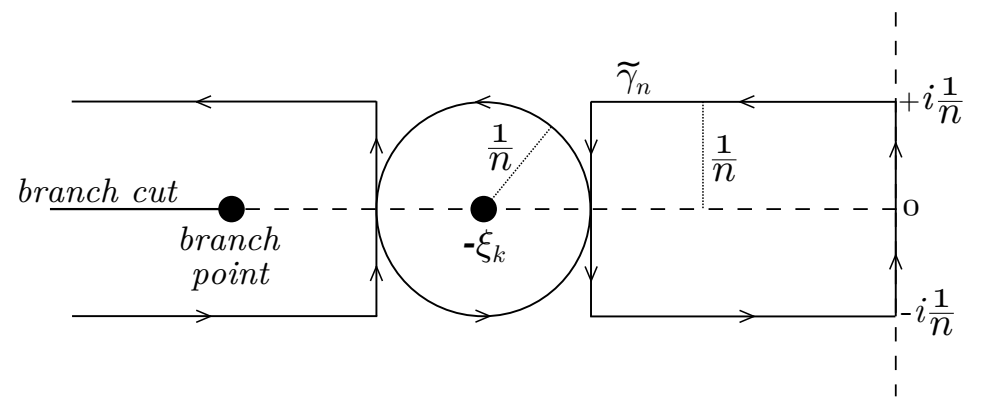

Figure 11: $\widetilde{\gamma}_{n}$ arc considered in the case where $\gamma(\xi)=-|\xi|$.

\section{A.2 About numerical approximations}

The state equation (35) is infinite-dimensional. To get numerical approximations, we consider $\mathcal{M}_{L}$ a sequence of $L$-dimensional spaces of atomic measures on suitable meshes $\left\{\xi_{l}^{L}\right\}_{l=1: L}$ on the variable $\xi ; L$-dimensional approximations $\nu^{L}$ of the $\gamma$-symbol $\nu$ are then defined in the sense of atomic measures, that is:

$$
\nu^{L}=\sum_{l=1}^{L} \nu_{l}^{L} \delta_{\xi_{l}^{L}}, \quad \nu_{l}^{L} \in \mathbb{C} .
$$

If $\cup_{L} \mathcal{M}_{L}$ is dense in the space of measures (that is, concretely, if $\cup_{L}\left\{\xi_{l}^{L}\right\}$ is dense in $\mathbb{R}$ ), then we can have (Montseny, 2005):

$$
\left\langle\nu^{L}, \psi\right\rangle \underset{L \rightarrow+\infty}{\longrightarrow}\langle\nu, \psi\rangle \forall \psi
$$

so, we have the following $L$-dimensional approximate state formulation of $H\left(\partial_{t}\right)($ with $\gamma$-symbol $\nu$ ):

$$
\left\{\begin{array}{l}
\partial_{t} \psi\left(t, \xi_{l}^{L}\right)=\gamma\left(\xi_{l}^{L}\right) \psi\left(t, \xi_{l}^{L}\right)+w(t), l=1: L \\
\psi\left(0, \xi_{l}^{L}\right)=0, l=1: L \\
{\left[H\left(\partial_{t}\right) w\right](t) \simeq \sum_{l=1}^{L} \nu_{l}^{L} \psi\left(t, \xi_{l}^{L}\right)}
\end{array}\right.
$$

The coefficients $\nu_{l}^{L}$ can be obtained by different ways:

- they can be deduced from the analytical expression of $\nu$ by the formula:

$$
\nu_{l}^{L}=\int \nu(\xi) \Lambda_{l}(\xi) d \xi
$$


with $\Lambda_{l}$ an interpolation function, for example the one given by:

$$
\Lambda_{l}(\xi)=\frac{\xi-\xi_{l-1}^{L}}{\xi_{l}^{L}-\xi_{l-1}^{L}} \mathbf{1}_{\left[\xi_{l-1}^{L}, \xi_{l}^{L}\right]}(\xi)+\frac{\xi_{l+1}^{L}-\xi}{\xi_{l+1}^{L}-\xi_{l}^{L}} \mathbf{1}_{]_{l}^{L}, \xi_{l+1}^{L}\right]}(\xi)
$$

If $\psi(t, \xi) \simeq \sum_{l=1}^{L} \psi\left(t, \xi_{l}^{L}\right) \Lambda_{l}(\xi)$, then we get $\langle\nu, \psi\rangle \simeq \sum_{l=1}^{L} \nu_{l}^{L} \psi\left(t, \xi_{l}^{L}\right)$.

- they can be numerically computed by resolution of the least squares problem (Montseny, 2005):

$$
\min _{\nu_{l}^{L} \in \mathbb{C}^{L}}\left\|\sum_{l=1}^{L} \frac{\nu_{l}^{L}}{p-\gamma\left(\xi_{l}^{L}\right)}-H(p)\right\|^{2},
$$

$\sum_{l=1}^{L} \frac{\nu_{l}^{L}}{p-\gamma\left(\xi_{l}^{L}\right)}$ being the transfer function of the operator defined by the finite dimensional state realization (40).

Depending on the method used, the obtained set of coefficients will be different, each one corresponding to one particular $\gamma$-symbol of the operator ${ }^{11}$. These coefficients are not necessarily positive. However, if $\nu$ is positive, and if the positivity of the coefficients $\nu_{l}^{L}$ is required, the first method will be preferred.

Most of non rational operators encountered in practice can be closely approximate with small $L$ (see for example (Montseny, 2004)), especially when the contour $\gamma$ satisfies a so-called "sectoral condition", for example when:

$$
\gamma(\xi)=|\xi| \mathrm{e}^{\mathrm{i} \operatorname{sign}(\xi)\left(\frac{\pi}{2}+\alpha\right)} \text { with } 0<\alpha \leqslant \frac{\pi}{2} .
$$

For the same precision, the numerical cost is then sometimes several orders lower than the one of standard integral quadratures.

\section{References}

Amhalhel G and Furmański P (1997) Problems of modeling flow and heat transfer in porous media. Journal of Power Technologies 85:5588.

\footnotetext{
${ }^{11}$ Recall indeed that a $\gamma$-symbol is not unique (see definition 3 ).
} 
Audounet J, Giovangigli V, and Roquejoffre JM (1998) A threshold phenomenon in the propagation of a point source initiated flame. Phys. D 121:295-316.

Baker G and Graves-Morris P (1996) Padé approximants. Cambridge University Press, UK, 59.

Biswal B and REN P-E and Held R J and Bakke S and Hilfer R (2009) Modeling of multiscale porous media. Image Analysis and Stereology 28:2334 .

Caputo M and Carcione JM (2011) Wave simulation in dissipative media described by distributed-order fractional time derivatives. Journal of $\mathrm{Vi}$ bration and Control 17(8):1121-1130.

Carmona P and Coutin L (1998) Fractional brownian motion and the markov property. Electron. Commun. Probab. 3:95-107.

Casenave C and Montseny G (2010) Introduction to diffusive representation. 4th IFAC Symposium on System, Structure and Control (SSSC 2010), Ancona (Italy), September 15-17.

Casenave C and Montseny E (2008) Dissipative state formulations and numerical simulation of a porous medium for boundary absorbing control of aeroacoustic waves. 17th IFAC World Congress, Seoul (Korea), July 6-11, pp.13432-13437.

Casenave C and Montseny E (2008) Formulation différentielle dissipative d'un modèle de paroi absorbante en aéroacoustique. Comptes rendus de l'Académie des Sciences (CRAS) - Mécanique, Vol.336, N4, pp.398-403.

Casenave C and Montseny E (2008) Time-local dissipative formulation and stable numerical schemes for a class of integrodifferential wave equations. SIAM J. Appl. Math. 341:1763-1783.

Chatellier L, Laumonier J and Gervais Y (2006) Active control of the aeroacoustics of cavity flows from the downstream edge. C. R. Mcanique Acad. Sci. Paris 334(4):259-265.

Degerli Y, Lavernhe F, Magnan P and Farre J (1999) Bandlimited 1/ $f^{\alpha}$ noise source. Electronics Letters 35:521-522. 
Garcia G and Bernussou J (1998) Identification of the dynamics of a lead acid battery by a diffusive model. ESAIM Proc. 5:87-98.

Gasser S (2003) Etude des propriétés acoustiques et mécaniques d'un matériau métallique poreux modèle à base de sphères creuses de nickel. $\mathrm{PhD}$ thesis, Institut National Polytechnique de Grenoble, France.

Kidner M R F and Hansen C H (2008) A comparison and review of theories of the acoustics of porous materials. International Journal of Acoustics and Vibration 13(3):112-119.

Lafarge D (1993)Propagation du son dans les matériaux poreux à structure rigide saturés par un fluide viscothermique. $\mathrm{PhD}$ thesis, Université du Mans, France.

Lai H and Luo K (2008) A conceptual study of cavity aeroacoustics control using porous media inserts. Flow, Turbulence and Combustion 80(3):375391.

Layton W J and Schieweck F and Yotov I (2002) Coupling fluid flow with porous media flow. SIAM Journal on Numerical Analysis 40(6):21952218.

Levadoux D and Michielsen B (1998) Analysis of a boundary integral equation for high-frequency Helmholtz problems. Proceedings of the Fourth International Conference on Mathematical and Numerical Aspects of Wave Propagation Golden, Colorado, June 1-5.

Levadoux D and Montseny G (2003) Diffusive formulation of the impedance operator on circular boundary for $2 \mathrm{D}$ wave equation. Six International Conference on Mathematical and Numerical aspects of Wave Propagation (Waves 2003), Jyväskylä, Finland, June 30-July 4.

Mainardi F, Mura A, Pagnini G and Gorenflo R (2008) Time-fractional diffusion of distributed order. Journal of Vibration and Control 17(9-10):2671290 .

Montseny G (2011) Diffusive wave-absorbing control: Example of the boundary stabilization of a thin flexible beam. Journal of Vibration and Control 18:1708-1721. 
Montseny G (2007) Diffusive representation for operators involving delays. In: Lecture Notes in Control and Inform. Sci. (J.-J. Loiseau \& J. Chiasson eds.) Applications of time-delay systems, pp. 217-232, Springer-Verlag.

Montseny G (2005) Représentation diffusive. Hermes Science, Paris.

Montseny G (2004) Simple approach to approximation and dynamical realization of pseudodifferential time-operators such as fractional one. IEEE Trans. Circuits Syst. II 51:613-618.

Mouyon P and Imbert N (2002) Identification of a 2D turbulent wind spectrum. Aerospace Science and Technology, 6:599-605.

Pride SR, Morgan FD and Gangi AF (1993) Drag forces of porous-medium acoustics. Phys. Rev. B 47:4964-4978.

Rege S D and Fogler H S (1987) Network model for straining dominated particle entrapment in porous media. Chemical engineering science 42(7):15531564 .

Rumeau A, Bidan P, Lebey T, Marchin L, Barbier B, and Guillemet S (2006) Behavior modeling of a $\mathrm{CaCu}_{3} \mathrm{Ti}_{4} \mathrm{O}_{12}$ ceramic for capacitor applications. IEEE Conference on Electrical Insulation and Dielectric Phenomena, Kansas City, Missouri USA, October 15-18.

Vogel H-J (2002) Topological characterization of porous media. Morphology of Condensed Matter 75-92.

Voronina N (1996) Improved empirical model of sound propagation through a fibrous material. Applied Acoustics 48(2):121-132. 